\title{
Adaptive Beamforming Based on QR Decomposition in Smart Antenna
}

\author{
Chengxin Xu, Ting Song, Xiangmei Li \\ Chengdu University of Information Technology, Chengdu, 610225, China \\ Email: xcx@cuit.edu.cn
}

Keywords: Smart Antenna, adaptive beamforming, matrix application

Abstract: This paper analyzes the key technology of smart antenna - the theories of beamforming and the definition of beamforming graph, and discussed how to realize the best beamformer through matrix theories. And through simulation on MATLAB, achieve the adaptive beamforming of antenna array.

\section{Research Overview}

Smart antenna is also called as adaptive antenna array, the difference between smart antenna and normal antenna is smart antenna receives signals by antenna array, and through changing weighting coefficient in every array element to choose the best beam and accomplish the best receiving ${ }^{11]}$. So adaptive beam forming is the key technology in smart antenna. How to implement 'adaptive' and 'best receiving' of the signal is the main problem to be solved. This paper will discuss adaptive beamforming and its implement by matrix theories.

\section{Theory model and beam forming algorithm}

Smart antenna consists of antenna array, A/D or D/A conversion, adaptive processing, beamforming 4 parts ${ }^{[2]}$. And its core part is beamforming network: through the weights from adaptive processing part, making dynamic adaptive weight to every array element, and at the same time forming the expected adaptive beam.

We assume that the baseband signal is:

$$
v(\mathrm{t})=\mathrm{s}(\mathrm{t}) \cdot \mathrm{H}(\theta) \cdot \mathrm{e}^{-j k \overrightarrow{r_{p}}}
$$

We set there are $\mathrm{M}$ antenna array elements, the array model of receiving signal can be expressed as:

$$
\mathbf{x}(\mathrm{t})=\left[\begin{array}{l}
x_{1}(\mathrm{t}) \\
x_{2}(\mathrm{t}) \\
\vdots \\
x_{M}(\mathrm{t})
\end{array}\right]=\left[\begin{array}{l}
\mathrm{H}_{1}(\theta) \cdot \mathrm{e}^{-j k \overrightarrow{r_{1}}} \\
\mathrm{H}_{2}(\theta) \cdot \mathrm{e}^{-j k \overrightarrow{r_{2}}} \\
\vdots \\
\mathrm{H}_{M}(\theta) \cdot \mathrm{e}^{-j k \overrightarrow{r_{M}}}
\end{array}\right] s(\mathrm{t}) \stackrel{\Delta}{=} \mathbf{a}(\theta, \phi) \mathrm{s}(\mathrm{t})
$$

Set the signal of unsuitable model as noise, we can get the model of multiple array signal model at n:

$$
\mathbf{x}(\mathrm{n})=\sum_{i=1}^{L} \mathbf{a}\left(\phi_{l}\right) \mathrm{s}_{l}(\mathrm{n})+\eta(\mathrm{n})
$$

We make the weight vector $\mathbf{w}=\left[w_{1}, w_{2}, \cdots w_{M}\right]^{T}$, the output signal of the array signal can be expressed as:

$$
\boldsymbol{y}(t)=\boldsymbol{w}^{H} \boldsymbol{x}(t)
$$


After weighting through some principles and algorithm, we can adjust the direction graph of array signal to one direction, and forming 'beam, ${ }^{[3]}$. We define beam responding as $\mathbf{w}^{H} \mathbf{a}$. The main idea of adaptive beamforming is changing the weighting value dynamically through some principles and algorithm, and through this way, we can get the signal of best quality. Then we will introduce one adaptive algorithm and the implement method of beamformer.

The sampling matrix inversion algorithm is raised by Reed. SMI is that by calculating autocorrelation matrix $\mathbf{r}_{x x}$ and cross correlation matrix $\boldsymbol{r}_{x d}$ in a period of time to get the estimation of weight vector. We take $\boldsymbol{R}_{x x}(n)$ and $\boldsymbol{r}_{x d}(n)$ to represent the estimate value of $\boldsymbol{R}_{x x}$ and $\boldsymbol{r}_{x d}$. We can get the estimating formula:

$$
\begin{aligned}
\mathbf{R}_{x x} & =\frac{1}{K} \sum_{k=1}^{K} \mathbf{x}(k) \mathbf{x}^{H}(k) \\
\mathbf{r}_{x d} & =\frac{1}{K} \sum_{k=1}^{K} d(k) \mathbf{x}(k)
\end{aligned}
$$

In the formula, $\mathrm{k}$ is the length of every data block.

It's very important in choosing the length of data block in SMI, it determines by the changing speed of channel ${ }^{[4]}$. When the speed is fast, in order to track the changing of channel, the update speed of weighting value must be high, so the block should be smaller. But when the channel changes slow, the data block can be larger.

Iterative algorithm of SMI can be summarized as follows:

(1) According to the data of current data block n, calculating the estimating value of autocorrelation matrix $\boldsymbol{R}_{x x}$ :

$$
\mathbf{R}_{x x}=\frac{1}{K} \sum_{k=1}^{K} \mathbf{x}(k) \mathbf{x}^{H}(k)
$$

(2) Calculating the estimating value of cross correlation matrix $\boldsymbol{r}_{x d}$ :

$$
\mathbf{r}_{x d}=\frac{1}{K} \sum_{k=1}^{K} d(k) \mathbf{x}(k)
$$

(3) Through the two estimating value, calculating the weighting vector:

$$
\boldsymbol{w}(n)=\boldsymbol{R}_{x x}^{-1}(n) \boldsymbol{r}(n)
$$

(4) When it comes to the next data block $n+1$, repeat step (1), and repeat all the steps until steady state.

The front ways of beamforming involves the computation of matrix inversion, it's very complex and the calculation is large, almost impossible to implement on electronic device ${ }^{[5]}$. Here, we discuss a practical method to realize it.

Assume the receiving noise in $\mathrm{n}$ data block has $k$ snapshot; we express the receiving data of array as:

$$
\begin{aligned}
X^{H} & =\left[x\left(\mathrm{n}_{1}\right), x\left(\mathrm{n}_{2}\right), \ldots, x\left(\mathrm{n}_{K}\right)\right] \\
& =\left[\begin{array}{llll}
x_{1}\left(\mathrm{n}_{1}\right) & x_{1}\left(\mathrm{n}_{2}\right) & \cdots & x_{1}\left(\mathrm{n}_{K}\right) \\
x_{2}\left(\mathrm{n}_{1}\right) & x_{2}\left(\mathrm{n}_{2}\right) & \cdots & x_{2}\left(\mathrm{n}_{K}\right) \\
\vdots & \vdots & \ddots & \vdots \\
x_{M}\left(\mathrm{n}_{1}\right) & x_{M}\left(\mathrm{n}_{2}\right) & \cdots & x_{M}\left(\mathrm{n}_{K}\right)
\end{array}\right]
\end{aligned}
$$

In the formula, $n_{k}$ represents the length of training correlation matrix. As for the sample matrix 
of length of $\mathrm{k}$, it can be expressed as:

$$
\hat{\mathbf{R}}=\frac{1}{K} \sum_{k=1}^{K} \mathbf{x}\left(\mathrm{n}_{k}\right) \mathbf{x}^{H}\left(n_{k}\right)=\frac{1}{K} \mathbf{X}^{H} \mathbf{X}
$$

After making QR decomposed to the matrix, we can get:

$$
\mathbf{X}=\mathbf{Q R}
$$

$\mathrm{Q}$ is a unitary matrix of $K \times M, \mathrm{R}$ is upper triangular matrix of $M \times M$ dimension. Define the lower triangular matrix $\mathbf{L} \stackrel{\Delta}{=} \frac{1}{\sqrt{K}} \mathbf{R}^{H}$, take it in $\hat{\mathbf{R}}=\frac{1}{K} \sum_{k=1}^{K} \mathbf{x}\left(\mathrm{n}_{k}\right) \mathbf{x}^{H}\left(n_{k}\right)=\frac{1}{K} \mathbf{X}^{H} \mathbf{X}$, we can get:

$$
\hat{\mathbf{R}}_{i+\eta}=\frac{1}{K} \mathbf{X}^{H} \mathbf{X}=\frac{1}{K} \mathbf{R}^{H} \mathbf{R}=\mathbf{L} \mathbf{L}^{H}
$$

As we know, the beamformer of MVDR principle is:

$$
\mathbf{w}_{o}=\frac{\hat{\mathbf{R}}_{i+\eta}^{-1} \mathbf{a}\left(\phi_{s}\right)}{\mathbf{a}^{H}\left(\phi_{s}\right) \hat{\mathbf{R}}_{i+\eta}^{-1} \mathbf{a}\left(\phi_{s}\right)}
$$

Because $\mathbf{Q}^{H} \mathbf{Q}=\mathbf{I}$, take it into (2.10), we can get:

$$
\mathbf{w}_{o}=\frac{\hat{\mathbf{R}}_{i+\eta}^{-1} \mathbf{a}\left(\phi_{s}\right)}{\mathbf{a}^{H}\left(\phi_{s}\right) \hat{\mathbf{R}}_{i+\eta}^{-1} \mathbf{a}\left(\phi_{s}\right)}=\frac{\mathbf{L}^{-H} \mathbf{L}^{-1} \mathbf{a}\left(\phi_{s}\right)}{\left|\mathbf{L}^{-1} \mathbf{a}\left(\phi_{s}\right)\right|^{2}}
$$

Above all, the implement steps of best beamformer are:

(1) Calculating the QR decomposition of matrix : $\mathbf{X}=\mathbf{Q R}$

(2) Normalization the upper triangular, and get the Choleskey factor $\mathbf{L}=(1 / \sqrt{K}) \mathbf{R}^{H}$.

(3) Get $\mathbf{z}_{1}$ from $\mathbf{L} \mathbf{z}_{1}=\mathbf{a}\left(\phi_{s}\right)$, get $\mathbf{z}_{2}$ from $\mathbf{L}^{H} \mathbf{z}_{2}=\mathbf{z}_{1}$

(4) Get the weighting vector of best beamformer $\mathbf{w}_{o}=\frac{\mathbf{z}_{2}}{\left\|\mathbf{z}_{2}\right\|^{2}}$

\section{The analysis of experiment}

We apply SMI to adaptive beamforming, and make simulation on MATLAB. Basic parameters: array elements: $\mathrm{n}=8$, the direction of useful signal $\theta_{s s}=30^{\circ}$, the direction of interference signal $\theta_{i i}=0^{\circ}$, snapshot number is 1000. Figure 1 is the amplitude response of adaptive beamforming based on SMI. Figure 2 is the beamforming graph of best beamformer.

From figure 1, we can see the main lobe directs the expected direction, and form hollow on interference signal direction. That is by using SMI algorithm, change the weighting value to make the signal gain on $\theta_{s s}=30^{\circ}$ be the largest, and on the interference direction $\theta_{i i}=0^{\circ}$, receiving gain is nearly 0 , therefore, finish the signal receiving. As the same, from figure 2 , we can see the result is almost the same. But what we should be aware that by best beamformer method, we can make calculation much easier, it makes the implement simpler, and save a lot. But we can also see that near the main lobe, there comes with one side lobe, it shows the Power leakage phenomenon. 


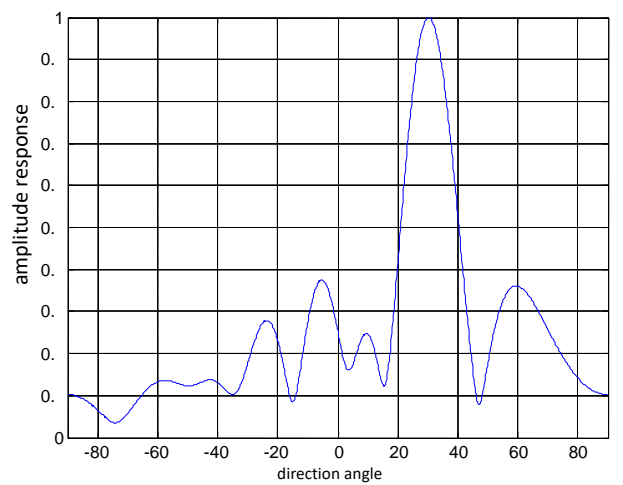

Fig.1. amplitude response based on SMI

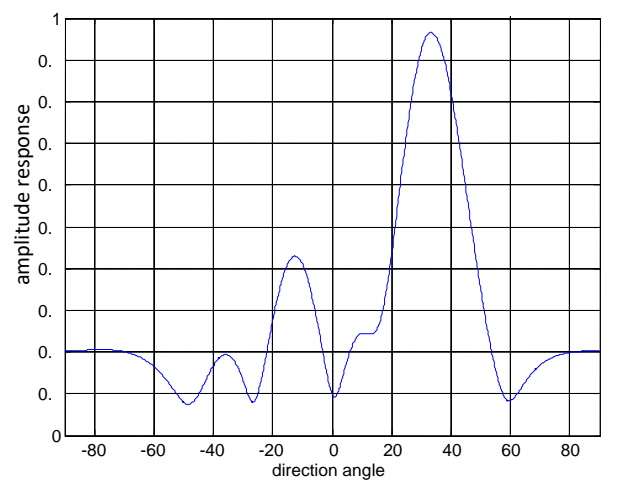

Fig.2. amplitude response of best beamformer

\section{Conclusions}

This paper discusses the adaptive algorithm in smart antenna and implementation method of best beamformer. The method has theoretical logic, and it has good feasibility in the project implementation, it is an important idea of the future development of smart antenna. At the same time, the algorithm uses a lot of matrix theory, which highlights the importance of matrix theory in smart antenna.

\section{References}

[1] Dogan M C, Mendel J M. Application of cumulant to array processing part: aperture extension and array calibration. IEEE Transactions on Signal Processing. 1995

[2] Song Y S, Kwon H M. Simple analysis of a simple smart antenna for CDMA wireless communication. Proc. IEEE Vehicular Technology Conf. 1999

[3] Rumiko Yonezawa, Kazufumi Hirata, Tetsuo Kirimoto. A Combination of Two Adaptive Algorithm SMI and CMA. IEEE Transactions on Signal Processing. 1998

[4] Chryssomallis M. Smart Antennas. IEEE Transactions on Antennas and Propagation. 2000

[5] C. Y. Kim, K. Lee,and Y. S. Cho.’ Adaptive Beamforming Algorithm for OFDM Systems with Antenna Arrays'. IEEE Transactions on Consumer Electronics. 2000 